\title{
Comunidade de fungos Agaricomycetes em diferentes sistemas florestais no noroeste do Estado do Rio Grande do Sul, Brasil: Floresta Estacional Decídua e monocultura de eucalipto
}

\author{
Diana Carla Lazarotto ${ }^{1,7}$, Jair Putzke ${ }^{2}$, Eliane Regina da Silva ${ }^{1}$, Lindamir Hernandez Pastorini ${ }^{3}$, \\ Carla Maria Garlet de Pelegrin ${ }^{4}$, Gerônimo Rodrigues Prado ${ }^{5}$ e Denise Cargnelutti ${ }^{6}$
}

Recebido: 26.07.2013; aceito: 20.01.2014

\begin{abstract}
Agaricomycetes fungi community at different forest systems in the northwest of Rio Grande do Sul State, Brazil: seasonal deciduous forest and eucalypt monoculture). Monocultures differ from native forests in relation to plant heterogeneity, variety of substrates, and soil humidity, possibly altering the diversity of organisms, such as the fungi. This study aimed to compare the richness, abundance, and composition of Agaricomycetes fungi in a native forest and in a eucalypt monoculture. The fungi were collected in a seasonal deciduous forest area and in a Eucalyptus grandis monoculture, in the northwest of Rio Grande do Sul State, Brazil. The abundance and richness of fungi were significantly lower in the eucalypt plantation than in the native forest and the species composition differed between the areas. Thus, it was concluded that the eucalypt monoculture negatively affects the Agaricomycetes fungi community.
\end{abstract}

Keywords: Basidiomycota, Eucalyptus grandis, native forest

RESUMO - (Comunidade de fungos Agaricomycetes em diferentes sistemas florestais no noroeste do Estado do Rio Grande do Sul, Brasil: Floresta Estacional Decídua e monocultura de eucalipto). As monoculturas diferem de matas nativas em relação à heterogeneidade vegetal, variedade de substratos e umidade do solo, podendo afetar a diversidade de organismos, tais como os fungos. O objetivo deste trabalho foi comparar a riqueza, abundância e composição de fungos Agaricomycetes em uma mata nativa e em uma monocultura de eucalipto. As coletas dos fungos foram realizadas em uma área de Floresta Estacional Decídua e em uma monocultura de Eucalyptus grandis, no noroeste do Estado do Rio Grande do Sul, Brasil. A abundância e riqueza de fungos na monocultura de eucalipto foram significativamente inferiores às encontradas na mata nativa, assim como a composição de espécies diferiu entre as áreas. Portanto, a monocultura de eucalipto afeta negativamente a comunidade de fungos Agaricomycetes.

Palavras-chave: Basidiomycota, Eucalyptus grandis, mata nativa

\section{Introdução}

A diversidade de espécies em ambientes nativos é geralmente maior do que em locais alterados. Isso porque os organismos diferem em seus requerimentos por habitat, e a resposta demográfica das populações é influenciada conforme as variações nas condições ambientais (Maurer 2009). Paisagens naturais apresentam poucas espécies comuns ou dominantes em cada grupo taxonômico e muitas espécies raras. No entanto, em paisagens alteradas pelas atividades humanas, intempéries climáticas e desastres naturais, há um aumento da dominância de espécies e uma redução da uniformidade entre elas, havendo poucas espécies com alta abundância relativa (Odum \& Barrett 2007).

1. Universidade Federal do Rio Grande do Sul, Instituto de Biociências. Av. Bento Gonçalves, 9500, Prédio 43433, Sala 213, Laboratório de Ecologia Química e Quimiotaxonomia, Agronomia, 91501-970 Porto Alegre, RS, Brasil

2. Universidade de Santa Cruz do Sul, Departamento de Biologia, Av Independência 2293, Universitário, 96815-900 Santa Cruz do Sul, RS, Brasil

3. Universidade Estadual de Maringá, Centro de Ciências Biológicas, Av. Colombo, 5790, Prédio G80, Sala 105, Campus Universitário, 87020-900 Maringá, PR, Brasil

4. Universidade Federal da Fronteira Sul, Campus Cerro Largo, Rua Major Antônio Cardoso, 590, Centro, 97900-000 Cerro Largo, RS, Brasil

5. Universidade Estadual do Rio Grande do Sul, Campus Cruz Alta, Rua Andrade Neves, 336, Centro, 98025-810 Cruz Alta, RS, Brasil

6. Universidade Federal da Fronteira Sul, Campus Erechim, Av. Dom João Hoffmann, 313, Fátima , 99700-000 Erechim, RS, Brasil

7. Autora para correspondência: diana.lazarotto@yahoo.com.br 
Em termos de diversidade biológica, há vários biomas que são considerados hotspots mundiais, áreas que abrigam grande diversidade e estão em iminência de destruição. Desses, destaca-se a Mata Atlântica que vem perdendo a sua cobertura original e sendo substituída por florestas exóticas plantadas, principalmente de espécies de Eucalyptus L'Hér. e Pinus L. (Câmara 2005). Monoculturas exóticas causam uma série de efeitos negativos aos mais variados grupos taxonômicos (Paritsis \& Aizen 2008). Isso decorre do fato de diferentes sistemas florestais diferirem quanto a fatores relacionados ao solo e vegetação (Claridge et al. 2000).

Dessa forma, os fungos também sofrem perdas de diversidade em monoculturas (Brown et al. 2006, Karstedt \& Stürmer 2008). Agaricomycetes é uma classe de fungos que agrupa fungos decompositores, ectomicorrízicos e parasitas (Hibbett 2006), incluindo 17 ordens do filo Basidiomycota (Kirk et al. 2008). Os fungos, em geral, dependem amplamente das variáveis ambientais, principalmente em relação à precipitação, umidade do ar e temperatura (Putzke \& Putzke 1998, Webster \& Weber 2007), bem como da disponibilidade de nutrientes e carbono no solo (Curlevski et al. 2010). Pesquisas que comparem diferentes sistemas florestais são raras, portanto, este estudo objetivou avaliar a riqueza, abundância e composição de fungos Agaricomycetes em uma área com Eucalyptus grandis, uma das espécies silviculturais mais cultivadas no país, e em uma área de Floresta Estacional Decídua, referida ao longo desse trabalho como mata nativa. Este trabalho destaca-se por trazer informações inéditas sobre a comunidade de macrofungos em uma área de mata nativa e outra área com Eucalyptus grandis, uma das espécies silviculturais mais cultivadas no país.

\section{Material e métodos}

Locais de estudo - As coletas de fungos da classe Agaricomycetes foram realizadas em uma área de mata nativa e em uma área com monocultura de eucalipto, ambas com aproximadamente 1,5 hectare, localizadas na região noroeste do Estado do Rio Grande do Sul. A primeira localiza-se no domínio da Mata Atlântica, onde a vegetação é caracterizada como Floresta Estacional Decídua (IBGE 2012). Essa área de mata nativa está situada no município de Caiçara $\left(27^{\circ} 18^{\prime} \mathrm{S}\right.$ e $53^{\circ} 24^{\prime} \mathrm{O}, 485 \mathrm{~m}$ de altitude ou 485 m.s.m. - como autores preferirem). A monocultura de eucalipto está distanciada em aproximadamente um quilômetro da mata nativa e localiza-se no município de Frederico Westphalen $\left(53^{\circ} 25^{\prime} \mathrm{O}\right.$ e $27^{\circ} 19^{\prime} \mathrm{S}, 500$ IDEM). Ela foi implantada há cinco anos em um local previamente destinado ao cultivo agrícola. O clima da região é caracterizado como subtropical subúmido, com temperatura média anual de $18,8^{\circ} \mathrm{C}$ e precipitação de $1787 \mathrm{~mm}$ (Maluf 2000). Os dados referentes à pluviosidade mensal da região de estudo no ano de 2010 foram disponibilizados pelo Instituto Nacional de Meteorologia (INMET) e utilizados em algumas das análises estatísticas.

Procedimento amostral - Os fungos Agaricomycetes foram coletados duas vezes em cada uma das estações (primavera, verão, outono e inverno) ao longo do ano de 2010, nas áreas com mata nativa e monocultura de eucalipto. Em cada local, foram selecionados aleatoriamente dez pontos de coleta, apresentando área de aproximadamente $8 \mathrm{~m}$ de diâmetro cada. Dessa forma, os pontos selecionados foram visitados duas vezes em cada estação do ano para a realização das coletas, totalizando oito coletas.

Depois de coletados, foram realizadas as observações macro e microscópicas, e a desidratação dos fungos segundo Putzke \& Putzke (1998). Para a identificação dos espécimes foram consultadas as seguintes bibliografias: Singer (1986), para fungos Agaricales; Ryvarden (1991) e Ryvarden (2004), para fungos poroides; Miller \& Miller (1988), para fungos gasteroides. A classificação taxonômica seguida foi a de Kirk et al. (2008) e a busca pelos autores das espécies foi feita no site Index Fungorum (www. indexfungorum.org). Somente espécimes de fungos Agaricomycetes maduros puderam ser identificados, os quais foram considerados nas análises dos dados. Os fungos coletados foram armazenados no Herbário HCB, na Universidade de Santa Cruz do Sul (UNISC), RS.

Análise dos dados - A riqueza, composição e abundância de fungos Agaricomycetes foram comparadas entre as áreas por testes de PERMANOVA univariada e multivariada, através de aleatorização, com 10.000 iterações (Pillar \& Orlóci 1996). A variação sazonal na riqueza e abundância de fungos foi estimada através do mesmo tratamento estatístico. O índice de diversidade de Shannon-Wiener foi calculado para as áreas e, posteriormente, submetido a teste de aleatorização para verificar a significância das diferenças entre os valores obtidos. A influência da pluviosidade sobre a riqueza e sobre a abundância de fungos foi testada através de regressão linear simples. 
Quando necessário, os dados foram transformados em $\log (\mathrm{x}+1)$, a fim de atingir os pressupostos do método paramétrico de regressão linear. Os softwares Multiv (Pillar 2006) e R (R Core Team 2013) foram utilizados para a realização dos testes estatísticos. As análises foram consideradas significativas quando $<0,05$.

\section{Resultados}

Neste estudo, foram encontrados 42 táxons de fungos da classe Agaricomycetes, pertencentes a 34 gêneros e 17 famílias (figura 1). O esforço amostral despendido resultou na coleta de 822 espécimes, 708 deles na mata nativa e 114 na monocultura de eucalipto. $\mathrm{Na}$ mata nativa, foram registrados 38 táxons, enquanto que na monocultura de eucalipto apenas oito. A abundância $\left(\mathrm{F}_{1,18}=5,38 ;<0,001\right)$ e a riqueza (Pseudo-F: 31,$20 ; p<0,001$ ) de fungos Agaricomycetes foram significativamente diferentes entre as áreas, sendo maiores na mata nativa em relação à monocultura de eucalipto. Da mesma forma, o valor de diversidade do índice de Shannon-Wiener foi maior para a mata nativa $H^{\prime}=1,55$ em relação à monocultura de eucalipto $\mathrm{H}^{\prime}=0,62$ e as áreas diferiram entre si quanto a esse parâmetro $(p<0,001)$.

A ordem Agaricales foi predominante tanto na mata nativa $(71,05 \%)$ quanto na monocultura de eucalipto $(62,5 \%)$. Na mata nativa, a família mais representativa foi Tricholomataceae, que apresentou seis táxons, seguida por Agaricaceae com cinco táxons. Na monocultura de eucalipto, as famílias tiveram igual representatividade. Na mata nativa, a maioria dos táxons apresentou baixa abundância relativa, enquanto que na monocultura esses valores foram altos (tabela 1). A composição de táxons diferiu entre as áreas $\left(\mathrm{F}_{1,18}=13,11 ; p=0,001\right)$, provavelmente porque apenas Geastrum fimbriatum, Psathyrella candolleana, Scleroderma sp. e Lycoperdon sp. ocorreram em ambas as áreas.

A abundância e riqueza de fungos Agaricomycetes apresentaram variação sazonal significativa na área de mata nativa (respectivamente, para abundância Pseudo-F: 8,04; $p<0,001$; e para riqueza Pseudo-F: 6,34; $p=0,009)$. Na monocultura de eucalipto, a abundância desses fungos também apresentou variação sazonal significativa (Pseudo-F: 1,37; $p<0,001$ ), já a riqueza

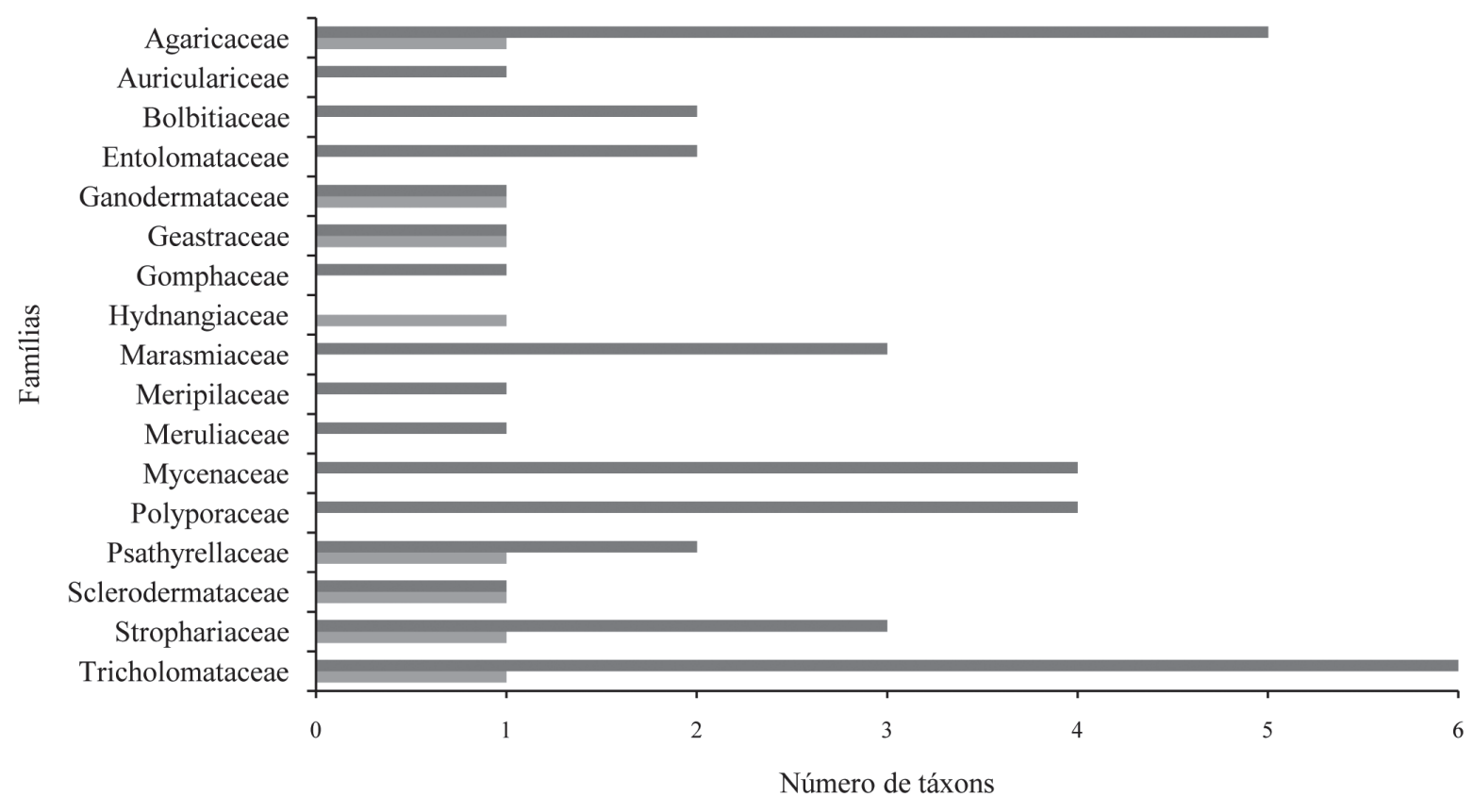

Figura 1. Número de táxons de fungos Agaricomycetes por família na área de floresta estacional decidual (mata nativa) e na monocultura de E. grandis, na região noroeste do Rio Grande do Sul- Brasil, em 2010. Mata Nativa, E. grandis.

Figure 1. Number of Agaricomycetes fungi taxa per family in areas of deciduous forest (native forest) and E. grandis monoculture, in the northwest region of Rio Grande do Sul- Brazil, in 2010. Native forest, $\square$ E. grandis. 
Tabela 1. Abundância relativa (\%) dos táxons de fungos Agaricomycetes coletados nas áreas de Floresta Estacional Decídua. MN: mata nativa; E: área com Eucaliptus grandis, na região noroeste do Estado do Rio Grande do Sul,Brasil, em 2010.

Table 1. Relative abundance (\%) of Agaricomycetes fungi taxa in deciduous forest. MN: native forest; E: Eucaliptus grandis, in the northwest region of Rio Grande do Sul State, Brazil, in 2010.

\begin{tabular}{|c|c|c|}
\hline Famílias/ Táxons & $\mathrm{MN}$ & $\mathrm{E}$ \\
\hline \multicolumn{3}{|l|}{ Agaricaceae Chevall. } \\
\hline Micropsalliota sp. & 0,85 & - \\
\hline Lepiota sp. & 1,41 & - \\
\hline Leucocoprinus sp. & 0,28 & - \\
\hline Leucocoprinus fragilissimus (Berk. \& M.A. Curtis) Pat. & 0,42 & - \\
\hline Lycoperdon sp. & 2,68 & 7,02 \\
\hline \multicolumn{3}{|l|}{ AURICULARIACEAE Fr. } \\
\hline Auricularia fuscosuccinea (Mont.) Henn. & 1,84 & - \\
\hline \multicolumn{3}{|l|}{ BOLBITIACEAE Singer } \\
\hline Bolbitius sp. & 0,71 & - \\
\hline Conocybe sp. & 1,13 & - \\
\hline \multicolumn{3}{|l|}{ ENTOLOMATACEAE Kotl. \& Pouzar } \\
\hline Clitopilus sp. & 0,14 & - \\
\hline Entoloma sp. & 0,71 & - \\
\hline \multicolumn{3}{|l|}{ GANODERMATACEAE Donk } \\
\hline Amauroderma sp. & & 26,31 \\
\hline Ganoderma sp. & 0,28 & - \\
\hline \multicolumn{3}{|l|}{ GEASTRACEAE Corda } \\
\hline Geastrum fimbriatum Fr. & 0,71 & 2,63 \\
\hline \multicolumn{3}{|l|}{ GOMPHACEAE Donk } \\
\hline Ramaria sp. & 21,75 & - \\
\hline \multicolumn{3}{|l|}{ HYDNANGIACEAE Gäum. \& C.W. Dodge } \\
\hline Laccaria fraterna (Sacc.) Pegler & & 17,54 \\
\hline \multicolumn{3}{|l|}{ MARASMIACEAE Roze $e x$ Kühner } \\
\hline Marasmiellus sp. 1 & 6,64 & - \\
\hline Marasmiellus sp. 2 & 2,82 & - \\
\hline Marasmius sp. & 19,49 & - \\
\hline \multicolumn{3}{|l|}{ MERIPILACEAE Jülich } \\
\hline Hydnopolyporus fimbriatus (Cooke) D.A. Reid & 15,40 & - \\
\hline \multicolumn{3}{|l|}{ MERULIACEAE P. Karst. } \\
\hline Cymatoderma caperatum (Berk. \& Mont.) D.A Reid & 0,14 & - \\
\hline MYCENACEAE Overeem & & - \\
\hline Filoboletus gracilis (Klotzsch ex Berk.) Singer & 5,65 & - \\
\hline Mycena sp. & 3,53 & - \\
\hline Mycena epipterygia (Scop) Gray & 3,95 & - \\
\hline Panellus pusillus (Pers. ex Lév.) Burds. \& O.K. Mill. & 0,99 & - \\
\hline \multicolumn{3}{|l|}{ POLYPORACEAE Fr. ex Corda } \\
\hline Panus velutinus (Fr.) Sacc. & 0,14 & - \\
\hline Polyporus sp. & 0,42 & - \\
\hline Polyporus ciliatus Fr. & 0,71 & - \\
\hline Polyporus tenuiculus (P. Beauv.) Fr. & 0,71 & - \\
\hline
\end{tabular}


Tabela 1 (continuação)

\begin{tabular}{lcc}
\hline Famílias/ Táxons & MN & E \\
\hline PSATHYRELACEAE Vilgalys, Moncalvo \& Redhead & 0,71 & - \\
$\quad$ Psathyrella sp. & 0,42 & 12,28 \\
Psathyrella candolleana (Fr.) Maire & & \\
SCLERODERMATACEAE Corda & 0,28 & 23,68 \\
$\quad$ Scleroderma sp. & & \\
STROPHARIACEAE Singer \& A.H. Sm. & 0,14 & - \\
Agrocybe sp. & 0,14 & - \\
Pholiota sp. & - & 3,51 \\
Psilocybe sp. & 0,85 & - \\
Gymnopilus sp. & \multicolumn{2}{|}{} \\
TRICHOLOMATACEAE R. Heim ex Pouzar & - & 7,02 \\
Clitocybe rivulosa (Pers.) P. Kumm. & 0,28 & - \\
Collybia sp. & 0,28 & - \\
Collybia dryophila (Bull.) P. Kumm. & 0,14 & - \\
Oudemansiella stefenii (Rick) Singer & 0,14 & - \\
Leucopaxillus brasiliensis (Rick) Singer \& A.H. Sm. & 2,82 & - \\
Neoclitocybe sp. & 0,28 & - \\
Neoclitocybe bissiseda (Bres.) Singer &
\end{tabular}

não teve variação sazonal significativa (Pseudo-F: 0,81; $p=0,31)$. Além da variação sazonal, foram confrontados os valores de precipitação mensal com a riqueza e abundância registradas, também mensalmente em ambas as áreas do estudo. Dessa forma, foi observado que a precipitação afetou significativamente a riqueza $\left(\mathrm{F}_{1,6}=5,55 ; p=0,05\right)$ (figura 2) e abundância de fungos Agaricomycetes na mata nativa $\left(\mathrm{F}_{1,6}=8,53 ; p=0,02\right)$ ao longo do ano de 2010. Já na monocultura, a precipitação não influenciou esses parâmetros (respectivamente, abundância $\mathrm{F}_{1,6}=0,76 ; p=0,42$; e riqueza $\mathrm{F}_{1,6}=0,092$; $p=0,77)$.

\section{Discussão}

As monoculturas não oferecem os mesmos recursos e não desempenham as mesmas funções de uma floresta nativa ao estabelecimento das espécies de fungos. Neste estudo, um fator determinante para a alta diversidade fúngica na mata nativa é, provavelmente, a variedade de substratos existentes nesse local. Lindblad (1998) relatou que substratos de madeira em decomposição são importantes para a diversidade de espécies de fungos que necessitam dos mesmos. $\mathrm{Na}$ monocultura de eucalipto não há toras de madeira caídas e acúmulo de material vegetal em decomposição como há na mata nativa, o que pode explicar em parte, os resultados encontrados nesse estudo. Outros autores também tentaram elucidar as razões pelas quais há diferenças na diversidade desses organismos entre ambientes diferentes. Karstedt \& Stürmer (2008) coletaram fungos da ordem Agaricales em plantações de Pinus elliottii Engelm. e em floresta ombrófila densa, obtendo maior diversidade na área nativa. Os autores acreditam que os resultados obtidos estejam relacionados à disponibilidade de matéria orgânica em decomposição, que é mais abundante e variada em ambientes naturais. Esses dados também corroboram a forte evidência encontrada por Lodge et al. (1995) de que a diversidade de fungos dos filos Ascomycota e Basidiomycota sejam influenciados pela diversidade de plantas hospedeiras, habitats e ecossistemas.

A composição de espécies de fungos também se mostrou significativamente diferente entre as áreas estudadas no presente trabalho. As características de cada ambiente, exótico e nativo, podem levar à seleção de espécies adaptadas a elas. Na monocultura de eucalipto, há prevalência de material vegetal dessa cultura depositado no solo, enquanto na área de mata nativa há disponibilidade de matéria orgânica variada em decomposição. Logo, quanto mais variados os substratos existentes em um ambiente, mais espécies de fungos podem se estabelecer (Lodge 1997). Assim, tal fato explica a baixa diversidade encontrada na monocultura de E. grandis. 


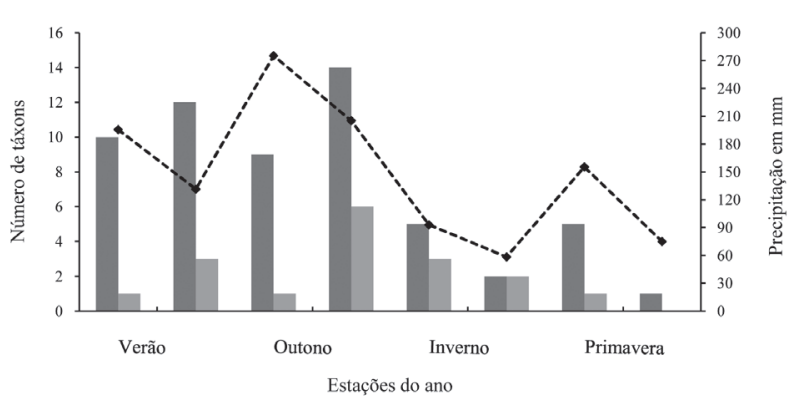

Figura 2. Variação da riqueza de táxons de fungos Agaricomycetes na área de Floresta Estacional Decídua (mata nativa) e na área com Eucalyptus grandis, e a precipitação registrada nos meses em que foram realizadas as coletas, na região noroeste do Estado do Rio Grande do Sul, Brasil, em 2010. Mata Nativa, — E. grandis, --๑-- Pluviosidade.

Figure 2. Taxa richness of Agaricomycetes fungi in areas of deciduous forest (native forest) and Eucalyptus grandis monoculture, and precipitation in the months when the fungi were collected, in the northwest region of Rio Grande do Sul State, Brazil, in 2010. Native Forest, $\square$ E. grandis, --^-- Precipitation.

A precipitação é um fator que possui influência direta na abundância e riqueza dos fungos Agaricomycetes na mata nativa. A diversidade de macrofungos, geralmente, é maior em lugares em que há intermediários a altos índices pluviométricos (Lodge et al. 1995). Assim como a precipitação influencia a umidade do solo, a abertura do dossel também pode atuar sobre essa variável. O dossel da monocultura de eucalipto é, em geral, mais aberto do que o dossel de florestas, proporcionando maior entrada de luminosidade e favorecendo maior evaporação de água do solo e de outros substratos que servem como microhabitat para os fungos.

Os resultados deste estudo mostram variações entre áreas com florestas naturais e exóticas. Ainda assim, muitos trabalhos defendem o uso de monoculturas como uma forma de amenizar os danos da fragmentação florestal de ambientes nativos, principalmente através do fornecimento de habitat para as espécies e conexão entre fragmentos remanescentes (Norton 1998, Ferris et al. 2000, Humphrey et al. 2000, Brockerhoff et al. 2008, O'Hanlon \& Harrrington 2012). Porém, cabe salientar que a maior parte desses estudos foi realizada em regiões temperadas, que apresentam baixa diversidade de espécies de plantas, mesmo em áreas nativas. Sendo assim, não há grande discrepância na diversidade desses sistemas florestais, e a implantação de monoculturas pode ser uma alternativa vantajosa. Já áreas neotropicais possuem elevada diversidade vegetal e qualquer homogeneização da paisagem ocasiona significativa depreciação na diversidade fúngica (Lodge et al. 1995).

A partir dos dados apresentados, fica evidente que a monocultura de eucalipto afeta negativamente a comunidade de fungos da classe Agaricomycetes e provavelmente esta conclusão pode ser válida também a outros grupos de fungos. A diferença na diversidade de fungos entre os sistemas florestais nativo e exótico deste estudo pode ser explicada principalmente pela heterogeneidade e diversidade de substratos encontrados em florestas nativas e a alta incidência de luminosidade através do estrato arbóreo da monocultura de E. grandis. Este trabalho de comparação traz conclusões relevantes e inéditas, tendo em vista os sistemas florestais estudados, acerca da conservação de espécies de fungos em ambientes nativos e plantados.

\section{Agradecimentos}

Ao Programa Institucional de Iniciação Científica (PIIC/Universidade Regional Integrada do Alto Uruguai e das Missões), pelo apoio financeiro fornecido ao primeiro Autor. Aos proprietários das áreas deste estudo, Sr. Filomeno Lipi e Sr. Jonides Trevisol, por terem permitido sua realização.

\section{Literatura citada}

Brockerhoff, E.G., Jactel, H., Parrotta, J.A., Quine, C.P. \&.Sayer, J.V. 2008. Plantation forests and biodiversity: oxymoron or opportunity? Biodiversity and Conservation 17: 925-951.

Câmara, I. G. 2005. Breve história da conservação da Mata Atlântica. In: C. Galindo-Leal \& I.G. Câmara (eds.). Mata Atlântica: Biodiversidade, Ameaças e Perspectivas. Belo Horizonte, Conservação Internacional e Fundação SOS Mata Atlântica, São Paulo, pp. 31-42.

Claridge, A.W., Barry, S.C., Cork, S.J. \& Trappe, J.M. 2000. Diversity and habitat relationships of hypogeous fungi. II. Factors influencing the occurrence and number of taxa. Biodiversity and Conservation 9: 175-199.

Curlevski, N.J.A., Xu, Z., Anderson, I.C. \& Cairney, J.W.G. 2010. Soil fungal communities differ in native mixed forest and adjacent Araucaria cunninghamii plantations in subtropical Australia. Journal of Soils Sediments 10:1278-1288.

Ferris, R., Peace, A.J. \& Newton, A.C. 2000. Macrofungal communities of lowland Scots pine (Pinus sylvestris L.) and Norway spruce (Picea abies (L.) Karsten.) plantations in England: relationships with site factors and stand structure. Forest Ecology and Management, 131: 255-267. 
Hibbett, D.S. 2006. A phylogenetic overview of the Agaricomycotina. Mycologia 9: 917-925.

Humphrey, J.W., Newton, A.C., Peace, A.J. \& Holden, E. 2000. The importance of conifer plantations in northern Britain as a habitat for native fungi. Biological Conservation 96: 241-252.

IBGE. 2012. Instituto Brasileiro de Geografia e Estatística. Manual técnico da vegetação brasileira. Série Manuais Técnicos em Geociências; 1. Rio de Janeiro.

Karstedt, F. \& Stürmer, S.L. 2008. Agaricales em áreas de Floresta Ombrófila Densa e plantações de Pinus no Estado de Santa Catarina, Brasil. Acta Botanica Brasilica 22: 1036-1043.

Kirk, P.M., Cannon, P.F., Minter, D.W. \& Stalpers, J.A. 2008. Ainsworth \& Bisby's Dictonary of the Fungi. CABI Publishing, United Kingdom.

Lindblad, I. 1998. Wood-inhabiting fungi on fallen logs of Norway spruce: relations to forest management and substrate quality. Nordic Journal of Botany 18: 243-255.

Lodge, D.J. 1997. Factors related to diversity of decomposer fungi in tropical forests. Biodiversity and Conservation 6: 681-688.

Lodge, D.J., Chapela, I., Samuels, G., Uecker, F.A., Desjardin, D., Horak, E., Miller Jr., O.K., Hennebert, G.L., Decock, C.A., Ammirati, J., Burdsall Jr, H.H., P., Kirk, M., Minter, D.W., Hailing, R., Laessøe, T., Mueller, G., Huhndorf, S., Oberwinkler, F., Pegler, D.N., Spooner, B., Petersen, R.H., Rogers, J.D., Ryvarden, L., Watling, R., Turnbull, E. \& Whalley, A.J.S. 1995. A Survey of Patterns of Diversity in NonLichenized Fungi. Mitteilungen der Eidgenössischen Forschungsanstalt für Wald, Schnee und Landschaft 70: 157-173.

Maluf, J.R.T. 2000. Nova classificação climática do Estado do Rio Grande do Sul. Revista Brasileira de Agrometeorologia 8: 141-150.

Maurer, B.A. 2009. Spatial patterns of species diversity in terrestrial environments. In: S.A. Levin (ed.). The Princeton Guide to Ecology. Princeton University Press, New Jersey, Princeton, pp. 464-473.
Miller, O.K. \& Miller, H.H. 1988. Gasteromycetes: morphological and developmental features. Eureka: Mad River, pp. 157.

Norton, D.A. 1998.Indigenous biodiversity conservation and plantation forestry: Options for the future. New Zealand Journal of Forestry 43: 34-39.

Odum, E.P. \& Barrett, G.W. 2007. O ecossistema. In: Odum, E.P. \& Barrett, G.W (eds). Fundamentos de ecologia. Thomson Learning, São Paulo, pp. 17-76.

O'Hanlon, R. \& Harrington, T.J. 2012. Macrofungal diversity and ecology in four Irish forest types. Fungal Ecology 5: 499-508.

Paritsis, J. \& Aizen, M.A. 2008. Effects of exotic conifer plantation on the biodiversity of understory plants, epigeal beetles and birds in Nothofagus dombeyi forests. Forest Ecology and Management 255: 1575-1583.

Pillar, V.D. 2006. MULTIV: Multivariate exploratory analisys, randomizating testing and boostraping resampling, users guide v. 2. 4. Universidade Federal do Rio Grande do Sul, Porto Alegre. http://ecoqua.ecologia. ufrgs.br/arquivos/Software/Multiv/MultivManual.pdf (acesso em 22.I.2012).

Pillar, V.D. \& Orlóci, L. 1996. On randomization testing in vegetation science: multifactor comparisons of relevé groups. Journal of Vegetation Science 7: 585-592.

Putzke, J. \& Putzke, M.T.L. 1998. Os Reinos dos Fungos. EDUNISC, Santa Cruz do Sul.

R Core Team. 2013. R: A language and environment for statistical computing. R Foundation for Statistical Computing, Vienna, Austria. http://www.R-project.org/ (acesso em 03.IV.2013).

Ryvarden, L. 1991. Genera of polypores: Nomenclature and taxonomy. Synopsis Fungorum 5: 1-363.

Ryvarden, L. 2004. Neotropical Polypores Part 1. Introduction, Ganodermataceae \& Hymenochaetaceae. Synopsis Fungorum 19: 1-229.

Singer, R. 1986. The Agaricales in Modern Taxonomy. 4 ed. Koeltz Scientific Books, Koenigstein.

Webster, J. \& Weber, R.W.S. 2007. Introduction to fungi. 3 ed. Cambridge University Press, New York. 